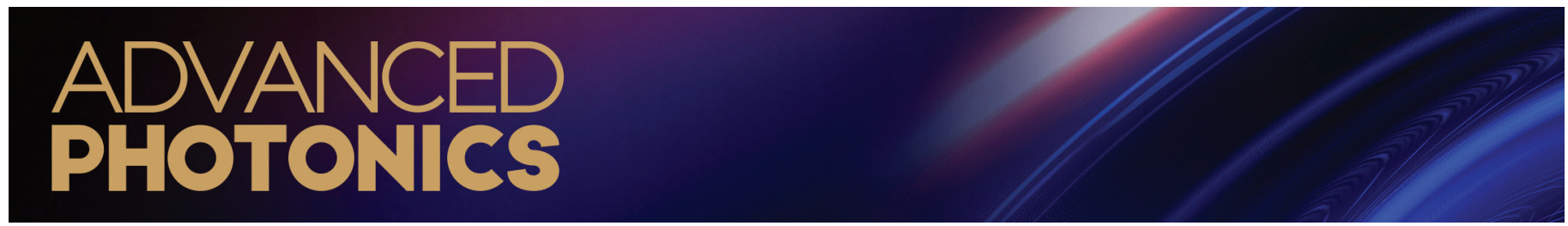

\title{
Resolving and weighing the quantum orbits in strong-field tunneling ionization
}

\author{
Jia Tan, ${ }^{\mathrm{a}, \mathrm{t}}$ Shengliang Xu, ${ }^{\mathrm{a}, \mathrm{t}}$ Xu Han, ${ }^{\mathrm{a}, \mathrm{t}}$ Yueming Zhou $\odot,{ }^{\mathrm{a}, \star}$ Min Li $\odot,{ }^{\mathrm{a}}$ Wei Cao, ${ }^{a}$ Qingbin Zhang, ${ }^{\mathrm{a}, \star}$ and Peixiang Lu ${ }^{\mathrm{a}, \mathrm{b}, \star}$ \\ ${ }^{a}$ Huazhong University of Science and Technology, School of Physics and Wuhan National Laboratory for Optoelectronics, Wuhan, China \\ ${ }^{b}$ Wuhan Institute of Technology, Hubei Key Laboratory of Optical Information and Pattern Recognition, Wuhan, China
}

\begin{abstract}
Tunneling ionization of atoms and molecules induced by intense laser pulses contains the contributions of numerous quantum orbits. Identifying the contributions of these orbits is crucial for exploring the application of tunneling and for understanding various tunneling-triggered strong-field phenomena. We perform a combined experimental and theoretical study to identify the relative contributions of the quantum orbits corresponding to the electrons tunneling ionized during the adjacent rising and falling quarter cycles of the electric field of the laser pulse. In our scheme, a perturbative second-harmonic field is added to the fundamental driving field. By analyzing the relative phase dependence of the signal in the photoelectron momentum distribution, the relative contributions of these two orbits are unambiguously determined. Our results show that their relative contributions sensitively depend on the longitudinal momentum and modulate with the transverse momentum of the photoelectron, which is attributed to the interference of the electron wave packets of the long orbit. The relative contributions of these orbits resolved here are important for the application of strong-field tunneling ionization as a photoelectron spectroscopy for attosecond time-resolved measurements.
\end{abstract}

Keywords: tunneling ionization; quantum orbits; photoelectron holography; attosecond electron dynamics.

Received Nov. 21, 2020; revised manuscript received Mar. 9, 2021; accepted for publication Apr. 29, 2021; published online May 25, 2021.

(C) The Authors. Published by SPIE and CLP under a Creative Commons Attribution 4.0 Unported License. Distribution or reproduction of this work in whole or in part requires full attribution of the original publication, including its DOI.

[DOI: 10.1117/1.AP.3.3.035001]

\section{Introduction}

Laser-induced tunneling ionization of atoms and molecules is a fundamental process in strong-field physics. It is the first step for various intriguing phenomena in attosecond science, such as high-order above-threshold ionization, ${ }^{1}$ high-order harmonic generation, ${ }^{2}$ and enhanced double/multiple ionization. ${ }^{3-5}$ Therefore, detailed understanding of the tunneling step is of fundamental importance for attosecond science. In the past decades, great efforts have been made to reveal the dynamics of strong-field tunneling. For instance, the questions of how long it takes the electron to tunnel through the potential barrier ${ }^{6-8}$ and when the tunneling electron appears at the outside of the barrier have been surveyed in depth. ${ }^{9-11}$ The features of the tunneling electron wave packet have also been widely studied. ${ }^{12-16}$

*Address all correspondence to Yueming Zhou, zhouymhust@ hust.edu.cn; Qingbin Zhang, zhangqingbin@ hust.edu.cn; Peixiang Lu, lupeixiang@ hust.edu.cn

tThese authors contributed equally to this work.
Theoretically, tunneling ionization of atoms and molecules in a strong laser field could be understood with the quantum-orbit (QO) model. ${ }^{17}$ In the QO model, the relationship between the ionization time and the final momentum of photoelectrons for each orbit was established. For plenty of the time-resolved measurements, the attosecond dynamics were extracted based on this concept. ${ }^{18-22}$ The concept of the QO model also provides a transparent picture for dynamics of various tunneling-triggered processes.

One intrinsic issue in the QO model of tunneling ionization is that for the same final momentum multiple quantum orbits are involved. ${ }^{17,23,24}$ The interferences of these orbits obscure the insights into the attosecond time-resolved information recorded by the photoelectrons. Thus, identifying the quantum orbits is a prerequisite for understanding the dynamics of the strong field processes and for the time-resolved measurements. Specifically, there are two types of orbits within one optical cycle for strongfield tunneling ionization, referred to as the long and short orbits, which correspond to the electrons tunneling being ionized 
during the adjacent ascending and descending quarter cycles of electric field, respectively. ${ }^{25,26}$ For the electron ionized during the descending quarter cycle (the long orbit), the electron could return back to the parent ion, resulting in high-order harmonic generation or high-energy photoelectrons, while the electron ionized during the ascending quarter cycle (the short orbit) usually does not contribute to these signals. Thus, for the measurements based on these signals, only the long orbit is involved. However, for the photoelectrons with low energy (lower than $2 U_{p}$, where $U_{p}$ is the ponderomotive energy), which has also been widely used to extract ultrafast electron dynamic information, ${ }^{18-22}$ both the long and short orbits contribute. Due to the Coulomb focusing effect after tunneling, it is accepted that the long orbit dominates over the short orbit for the signals in the photoelectron momentum distribution (PEMD), and thus temporal information is usually obtained based on the assumption that the long orbit contributes exclusively. Obviously, this approximation is questionable. In a recent experiment, it has been shown that the extracted ionization time with this approximation deviates significantly from the theoretical prediction. ${ }^{27}$ Recently, with the scheme of an orthogonal two-color field, the relative contributions of the long and short orbits were theoretically estimated ${ }^{28,29}$ In that scheme, the relative contributions were obtained with two time-to-momentum maps of the short and long orbits. However, the effect of the Coulomb interaction on the ionization time in the orthogonal two-color field cannot be measured, ${ }^{30}$ and thus the maps used there are calculated based on a strong-field approximation where the ionization time is inaccurate. Instead, the parallel two-color field could record the Coulomb effect on the ionization time, and it can be employed to identify the contributions of the long and short orbits. ${ }^{30} \mathrm{So}$, in this study, we perform a combined experimental and theoretical study to determine the relative contributions of the long and short orbits in tunneling ionization with the parallel two-color field. We monitor the photoelectron yield as a function of the relative phase at each momentum of the PEMD. This relative phase dependence of the signal is determined by the ionization time, and thus it is different for the long and short orbits. With the Coulomb-corrected strong-field approximation (CCSFA), we calculate the relative phase dependences of the photoelectron yields for the long and short orbits separately. By comparing the measured relative phase dependence of the photoelectron yield with these theoretical data, the individual contributions of the long and short orbits in tunneling ionization are qualitatively determined. The obtained results show that their contributions depend on both the longitudinal and transverse momenta, and in certain regions of the PEMD, the contributions from the short orbit could be even higher than that of the long orbit. This information is crucial for accurately determining the ionization time of photoelectron in the PEMD, which is the basis of the time-resolved measurements with the photoelectron from tunneling.

\section{Methods}

In our scheme, the parallel two-color field consists of a strong $800 \mathrm{~nm}$ field and a much weaker second harmonic (SH) field, and it is polarized along the $x$ direction. In the experiment (see the Supplemental Materials for details about our experiment and the numerical calculation with CCSFA, which includes Ref. 31), the SH pulse was produced by frequency doubling a femtosecond laser pulse ( $25 \mathrm{fs}, 800 \mathrm{~nm}, 1 \mathrm{kHz})$ from a Ti:sapphire laser system using a $300 \mu \mathrm{m}$-thick $\beta$-barium-borate crystal. The parallel two-color pulse was generated in a phase- locked Mach-Zehnder interferometer scheme. The relative phase $\varphi$ was finely controlled by the phase-locking system. ${ }^{32,33}$ The parallel two-color pulse was tightly focused onto a supersonic gas jet of Ar by a concave silver mirror $(f=15 \mathrm{~cm})$ in an ultrahigh vacuum chamber of a velocity map imaging setup. ${ }^{34}$ The PEMDs were then recorded as a function of the phase delay between the FM and SH fields, using a step size of $0.1 \pi$. The peak intensities of FM and SH fields were $\sim 1.2 \times 10^{14}$ and $\sim 0.3 \times 10^{11} \mathrm{~W} / \mathrm{cm}^{2}$, respectively. The relative phase of the two-color field is calibrated by comparing the photoelectron yield obtained from the experiment with that obtained by solving the time-dependent Schrödinger equation (see the Supplemental Materials for details).

\section{Results and Discussion}

Figures 1(a)-1(c) show the measured two-dimensional PEMDs for the parallel two-color field with relative phases $\varphi=0,0.5 \pi$, and $\pi$, respectively. As mentioned above, numerous quantum orbits contribute to the PEMDs, giving rise to the interference structures such as the above-threshold ionization rings ${ }^{35}$ and the spider-like holographic pattern. ${ }^{18,36}$ The interference of the long and short orbits that we will focus on is not visible due to the laser focal volume effect. This does not affect the determination of their relative contributions in this study. In our experiment, the laser intensity of the SH field is three orders of magnitude weaker than that of the FM field, and thus its perturbation on the PEMDs is tiny. To reveal the effect of the weak SH more clearly, we calculate the normalized difference (ND) of the PEMDs. The ND is defined as

$$
\mathrm{ND}(\mathbf{p} ; \varphi)=\frac{Y(\mathbf{p} ; \varphi)-Y_{\mathrm{avg}}(\mathbf{p})}{Y(\mathbf{p} ; \varphi)+Y_{\mathrm{avg}}(\mathbf{p})},
$$

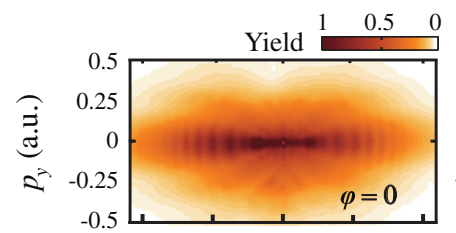

(a)

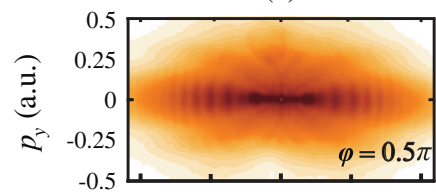

(b)

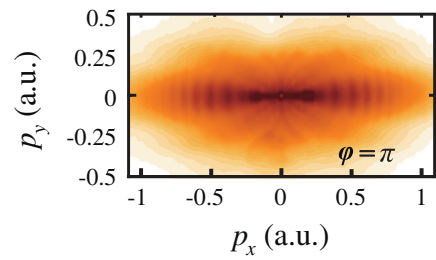

(c)

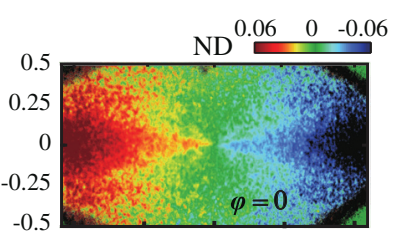

(d)

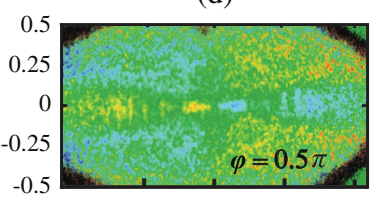

(e)

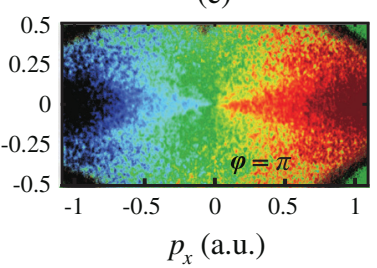

(f)
Fig. 1 (a)-(c) The experimentally measured PEMDs for strong-field tunneling ionization of Ar by the parallel two-color $(800 \mathrm{~nm}+400 \mathrm{~nm})$ field with relative phase $\varphi=0,0.5 \pi$, and $1.5 \pi$, respectively. The laser intensities of the FM and $\mathrm{SH}$ fields are $1.2 \times 10^{14}$ and $0.3 \times 10^{11} \mathrm{~W} / \mathrm{cm}^{2}$, respectively. (d)-(f) NDs for the distributions in (a)-(c) (see text for details). 
where $Y(\mathbf{p} ; \varphi)$ is the PEMD at the relative phase of $\varphi$, and $Y_{\text {avg }}(\mathbf{p})$ denotes the average distribution of the PEMDs for $\varphi$ ranging from 0 to $2 \pi$. Figures $1(\mathrm{~d})-1$ (f) show the NDs at $\varphi=0,0.5 \pi$, and $\pi$, respectively. The relative phase dependence of the photoelectron yields can be clearly seen. For instance, the signals at $p_{x}>0$ are reduced and enhanced at $\varphi=0$ and $\varphi=\pi$, respectively. For the signals at $p_{x}<0$, the situation is reversed.

To quantitatively characterize the relative phase dependence of the signal, we further analyze the NDs employing the phaseof-the-phase spectroscopy, ${ }^{37,38}$ which has been proposed to reveal the different orbits in strong-field ionization. ${ }^{39-41}$ In this method, the higher-order Fourier component of NDs is very small, and thus it is canceled out safely here. Figure 2(a) shows an example of the signal at the momentum $\mathbf{p}=(-0.6,0.1)$ a.u. as a function of the relative phase. It suggests that the photoelectron yield on the relative phase $\varphi$ at momentum $\mathbf{p}$ can be described as

$\mathrm{ND}(\mathbf{p} ; \varphi)=P(\mathbf{p}) \cos \left[\varphi-\varphi_{m}(\mathbf{p})\right]$,

where $P(\mathbf{p})$ characterizes the amplitude of the modulation, and it is irrelevant in this study (we drop this factor in the analysis below). $\varphi_{m}(\mathbf{p})$ indicates the relative phase where $\mathrm{ND}(\mathbf{p} ; \varphi)$ maximizes. The optimal phase $\varphi_{m}$ depends on both the transverse and longitudinal momenta, as shown in Figs. 2(b) and 2(c). We perform this analysis to each momentum in the PEMDs, and the obtained $\varphi_{m}$ is shown in Fig. 2(d). To see the momentum dependence of the phase $\varphi_{m}(\mathbf{p})$ more clearly, three cuts at $p_{x}=-0.4,-0.5$, and -0.6 a.u. from Fig. 2(d) are shown in Fig. 2(e). It is shown that the phase oscillates between $1.5 \pi$ and $1.9 \pi$ as $p_{y}$ changes, and when $p_{x}$ increases from -0.6 to -0.4 a.u. the phase shifts to smaller values. In the following, we will show that this quantity encodes information about the relative contributions of the long and short orbits in tunneling ionization.

In the two-color field, it is intuitive that the photoelectron yield at momentum $\mathbf{p}$ maximizes at the relative phase where the instantaneous electric field at ionization maximizes. ${ }^{30}$ Thus, the optimal phase is determined by the ionization time. However, the situation is more complex when multiple orbits contribute to the signal in the PEMDs. In Fig. 3(a), we illustrate the two orbits (the long and short orbits) within one optical cycle that contribute to the PEMDs. Due to the different ionization times of the long and short orbits, the photoelectron yields corresponding to these two orbits should maximize at different relative phases. The experimentally measured optimal phase provides us with a way to assess the contributions of these two orbits.

To this end, the key information is the optimal phase for each orbit. In the previous studies with a scheme of an orthogonal two-color field, this information is obtained with the strong-field approximation, where the Coulomb effect on the tunneling ionized electron is neglected. ${ }^{28,29}$ For more accurate optimal phases, it is necessary to include the effect of Coulomb interaction on the relationship of the ionization time and final momentum. This is crucial for our parallel two-color field. Thus, we calculate the modulation of the photoelectron yield as a function of the relative phase $\varphi$ at each momentum with the CCSFA (see Supplemental Materials for details), the accuracy of which has been well confirmed. ${ }^{42-45}$ Figures 3(b) and 3(c) show the calculated NDs as functions of the relative phases for the long and short orbits, respectively. Here, we display the signals at zero

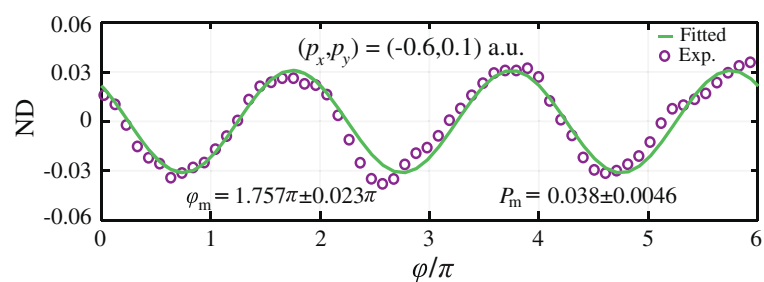

(a)

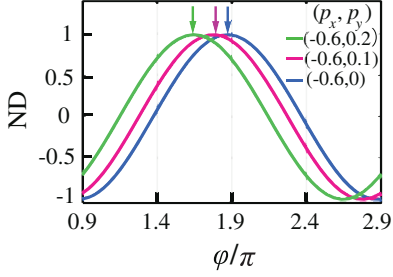

(b)

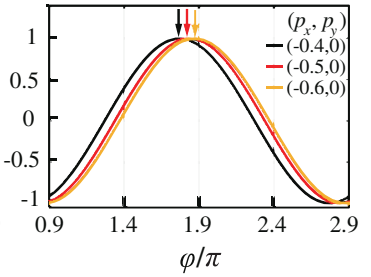

(c)

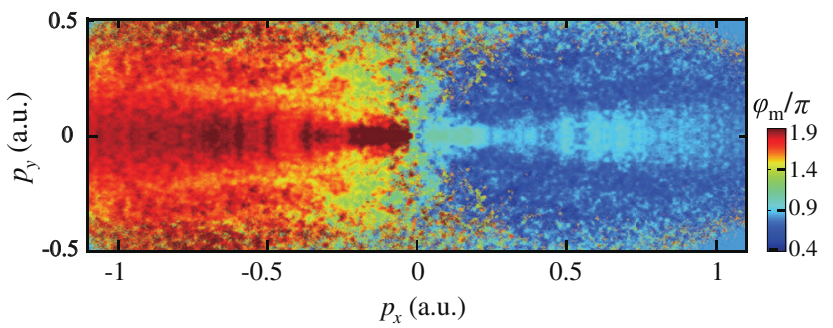

(d)

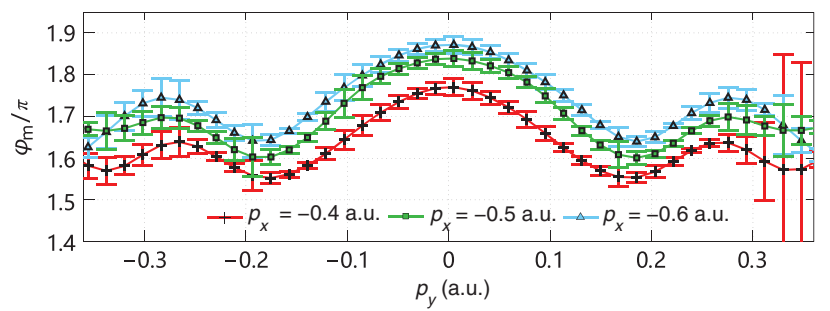

(e)

Fig. 2 (a) The ND as a function of $\varphi$ for the momentum $\left(p_{x}, p_{y}\right)=$ $(-0.6,0.1)$ a.u. The open circles show the experimental data and the green curve shows the fitted results. (b) The fitted ND by Eq. (2) as a function of $\varphi$ for the momentum $\left(p_{x}, p_{y}\right)=(-0.6,0)$ (blue curve), $(-0.6,0.1)$ (purple curve), and $(-0.6,0.2)$ a.u. (green curve). The data are normalized such that the maximum of each curve is unity. (c) Same as (b) but for $\left(p_{x}, p_{y}\right)=(-0.4,0)$ (black curve), $(-0.5,0)$ (red curve), and $(-0.6,0)$ a.u. (yellow curve). (d) The optimal phase $\varphi_{m}$ in the region of $p_{x} \in[-1.1,1.1]$ a.u. and $p_{y} \in[-0.5,0.5]$ a.u. (e) Cuts of $\varphi_{m}$ at $p_{x}=-0.4$ a.u. (red crosses), -0.5 a.u. (green squares), and -0.6 a.u. (blue triangles). The error bars show the $95 \%$ confidence interval in fitting.

transverse momentum. It is shown that for the long orbit the phase where the ND maximizes varies from $1.85 \pi$ to $2.05 \pi$ as $p_{x}$ increases from -1.0 to -0.2 a.u., and for the short orbit, the phase changes from $1.45 \pi$ to $1.25 \pi$. For each momentum, we repeat our calculation in the PEMD and extract the optimal phase $\varphi_{m}$. The results of the long and short orbits are shown in Figs. 3(d) and 3(e), respectively.

In the measured PEMDs, both the long and short orbits contribute, and the relative phase $\varphi_{m}$ should locate within the phases window determined by the long and short orbits. Two examples are shown in Figs. 4(a) and 4(b). For $\mathbf{p}=(-0.5,0)$ a.u. in Fig. 4(a), the optimal phases $\varphi_{m}$ determined by the long and 


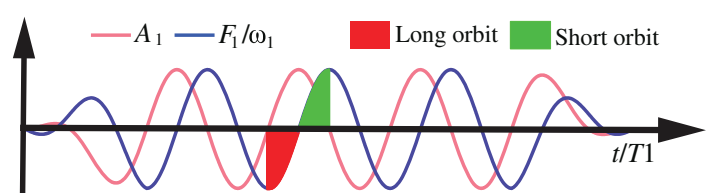

(a)

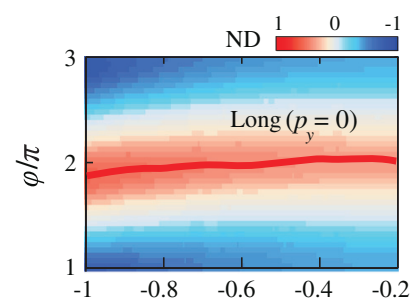

(b)

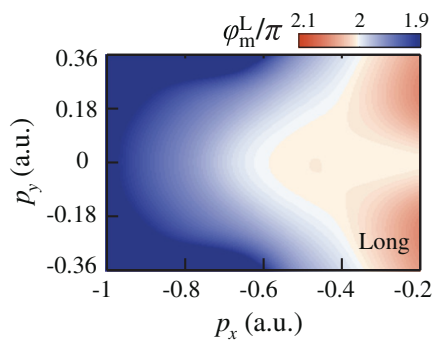

(d)

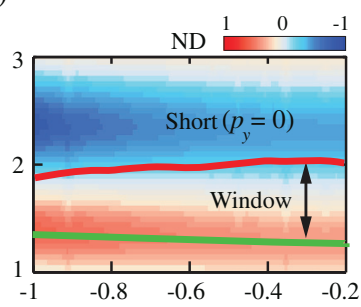

(c)

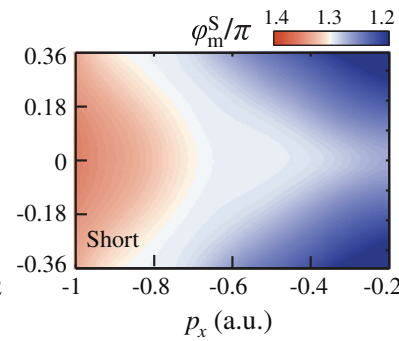

(e)
Fig. 3 (a) Illustration of the ionization times of the long and short orbits in strong-field tunneling ionization. The long and short orbits correspond to the ionization events, where the electron is released at the falling and rising edges of electric field, respectively. The blue curve indicates the electric field of the FM field and the red curve shows its vector potential. (b) The ND(p; $\varphi)$ for the long orbit with transverse momentum $p_{y}=0$, calculated by CCSFA. The solid red curve indicates the optimal phase $\varphi_{m}^{\mathrm{L}}$. (c) The same as (b) but for the short orbit. The black arrow denotes the phase window formed by $\varphi_{m}^{\mathrm{L}}$ and $\varphi_{m}^{\mathrm{S}}$. (d) The optimal phase $\varphi_{m}^{\mathrm{L}}$ of the long orbit for $p_{x} \in[-1,-0.2]$ a.u. and $p_{y} \in[-0.36,0.36]$ a.u. (e) The same as (d) but for the short orbit.

short orbits are $2.05 \pi$ and $1.3 \pi$. As expected, the optimal phase of the measured data is located between these values, and it is closer to the value of the long orbit, implying that the long orbit has the dominant contributions. For $\mathbf{p}=(-0.5,0.2)$ a.u., the phase of the experimentally measured data shifts toward the short orbit, meaning that the contributions of the short orbit increase.

The measured phase dependence of the ND at each momentum can be formulated as (see Supplemental Materials for details)

$\mathrm{ND}(\mathbf{p} ; \varphi)=\alpha \cos \left[\varphi-\varphi_{m}^{\mathrm{L}}(\mathbf{p})\right]+\beta \cos \left[\varphi-\varphi_{m}^{\mathrm{S}}(\mathbf{p})\right]$,

where $\alpha$ and $\beta$ denote the corresponding coefficients for the contributions of long and short orbits, respectively. $\varphi_{m}^{\mathrm{L}}(\mathbf{p})$ and $\varphi_{m}^{\mathrm{S}}(\mathbf{p})$ are the optimal phases for the long and short orbits [as shown in Figs. 3(d) and 3(e)], respectively. Note that in Eq. (3) the interference term of the long and short orbits has been dropped because in experiment this type of interference is not visible, as shown in Fig. 1. Fitting the experimental data with Eq. (3), the coefficients $\alpha$ and $\beta$ are obtained. For the data in Figs. 4(a) and 4(b), the obtained relative contributions $\alpha / \beta$ of

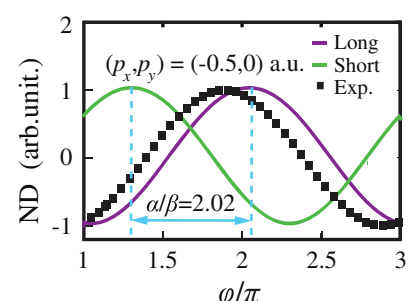

(a)

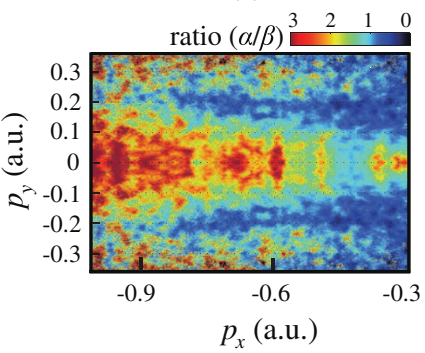

(c)

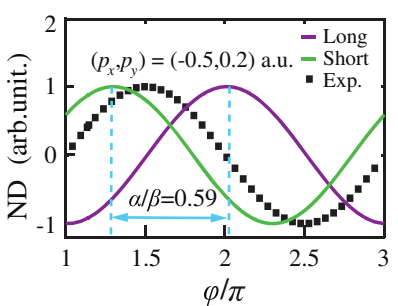

(b)

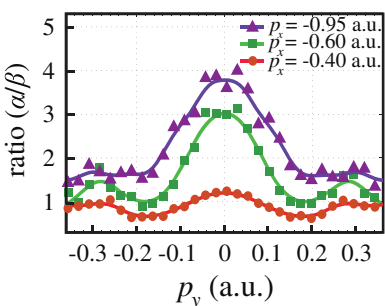

(d)
Fig. 4 (a) The ND at $\left(p_{x}, p_{y}\right)=(-0.5,0)$ a.u. as a function of $\varphi$. The solid green and purple curves show the theoretical results of the signal from the long and short orbits, respectively. The black squares represent the experimental data, where both the long and short orbits contribute. The dashed blue lines indicate the optimal phases $\varphi_{m}^{\mathrm{L}}$ and $\varphi_{m}^{\mathrm{S}}$, and the blue arrow denotes their phase window. (b) The same as (a) but for $\left(p_{x}, p_{y}\right)=(-0.5,0.2)$ a.u. (c) The ratios $\alpha / \beta$ extracted from the experimental data. (d) The cuts of $\alpha / \beta$ at $p_{x}=-0.4$ (red circles), -0.6 (green squares), and -0.95 a.u. (purple triangles), respectively.

the two orbits are 2.02 and 0.59 , respectively. We perform this fitting to each momentum in the PEMDs with $\varphi_{m}^{\mathrm{L}}(\mathbf{p})$ and $\varphi_{m}^{\mathrm{S}}(\mathbf{p})$, and the obtained ratio $\alpha / \beta$ is shown in Fig. 4(c). The result indicates that the relative contributions of the long and short orbits sensitively depend on both the longitudinal and transverse momenta. For the distribution along the $p_{x}$ axis, the long orbit has the main contributions, while for the distribution around $p_{y}=$ \pm 0.2 a.u., the ratio $\alpha / \beta$ is very close to unity, meaning that the long and short orbits have comparable contributions to the photoelectron yield. This behavior is due to the holographic interference in the long orbit. For the long orbit, the electron can be driven back to the parent ion after tunneling ionization, and the electron wave packet is split into two pathways. One suffers a near-forward recollision and the other without recollision. The two pathways give rise to the interference in the PEMDs. ${ }^{18,36} \mathrm{At}$ the interference maxima or minima, the contributions of the long orbit are enhanced or suppressed, leading to the modulation of the ratio $\alpha / \beta$ with the transverse momentum.

In the time-resolved measurements using photoelectrons from strong-field tunneling ionization, a map of time-tomomentum is necessary for extracting attosecond dynamics. In previous studies, this map was established using the long orbit, because it was assumed that the contributions of the long orbit dominate the signal in the PEMDs. ${ }^{27}$ Due to the constructive holographic interference, it is true that for the signal in the PEMD along the laser polarization direction, the contribution of the long orbit is larger than that of the short orbit. However, the ratio is only about four times higher, as shown in Fig. 4(c). Neglecting the contributions of the short orbit leads to the inaccuracy of the time-to-momentum map. Specifically, at the minimum of the holographic interference, the contributions of 
the short orbit could be even larger than that of the long orbit, as shown in Fig. 4(d). So, the time-resolved studies of revealing attosecond dynamics with the time-to-momentum map solely based on the long orbit are invalid. The inaccuracy of this map induced by the multiple orbits has also been realized in a very recent study. ${ }^{41}$ Furthermore, we should mention that in our scheme the multiple cycle pulses are used. For the multiple cycle pulses, the electric field during the adjacent rising and falling quarter cycles is the same, and the relative contributions of these two orbits are the same from cycle to cycle. However, in the few cycle pulses, the electric field of the adjacent quarter cycles is different, and the relative contributions of these orbits are different from cycle to cycle. Thus, for the few cycle pulses the scheme will break down.

\section{Conclusions}

We have experimentally determined the contributions of the long and short orbits in strong-field tunneling ionization. Due to the holographic interference of the electrons of the long orbits, the relative contributions of the long and short orbits depend on both the transverse and longitudinal momenta. At the holographic interference minimum, the contributions of the long orbit are even less than that of the short orbit. In previous studies, the ionization time of the photoelectron is usually determined based on the assumption that the long orbit dominates the photoelectron yields. Our results indicate this assumption is invalid, and it will induce inaccuracy in determining the time-to-momentum map. Our study provides a complete map to quantify the relative contributions of different quantum orbits for the signal at each momentum in the PEMDs. This information is necessary for establishing accurate correspondence between the ionization time and the final photoelectron momentum in the PEMDs, and thus it is important for the application of strong-field tunneling ionization as a photoelectron spectroscopy to probe atom and molecule dynamics in attosecond scale.

\section{Acknowledgments}

This work was supported by the National Key Research and Development Program of China (Grant No. 2019YFA0308300) and the National Natural Science Foundation of China (Grant Nos. 11874163, 12021004, 11627809, 11934006).

\section{References}

1. W. Becker et al., "Above-threshold ionization: from classical features to quantum effect," Adv. At. Mol. Opt. Phys. 48(C), 35-98 (2002).

2. F. Krausz and M. Ivanov, "Attosecond physics," Rev. Mod. Phys. 81(1), 163-234 (2009).

3. T. Weber et al., "Correlated electron emission in multiphoton double ionization," Nature 405(6787), 658-661 (2000).

4. Y. Zhou et al., "Asymmetric electron energy sharing in strong-field double ionization of helium," Phys. Rev. A 82(5), 053402 (2010).

5. W. Becker et al., "Theories of photoelectron correlation in laserdriven multiple atomic ionization," Rev. Mod. Phys. 84(3), 10111043 (2012).

6. P. Eckle et al., "Attosecond ionization and tunneling delay time measurements in helium," Science 322(5907), 1525-1529 (2008).

7. N. Camus et al., "Experimental evidence for quantum tunneling time," Phys. Rev. Lett. 119(2), 023201 (2017).

8. U. S. Sainadh et al., "Attosecond angular streaking and tunnelling time in atomic hydrogen," Nature 568(7750), 75-77 (2019).
9. D. Shafir et al., "Resolving the time when an electron exits a tunnelling barrier," Nature 485(7398), 343-346 (2012).

10. J. Zhao and M. Lein, "Determination of ionization and tunneling times in high-order harmonic generation," Phys. Rev. Lett. 111(4), 043901 (2013).

11. J. Tan et al., "Determination of the ionization time using attosecond photoelectron interferometry," Phys. Rev. Lett. 121(25), 253203 (2018).

12. M. Ivanov et al., "Anatomy of strong field ionization," J. Mod. Opt. 52(2-3), 165-184 (2005).

13. M. Meckel et al., "Laser-induced electron tunneling and diffraction," Science 320(5882), 1478-1482 (2008).

14. A. N. Pfeiffer et al., "Attoclock reveals natural coordinates of the laser-induced tunnelling current flow in atoms," Nat. Phys. 8(1), 76-80 (2012).

15. M. Meckel et al., "Signatures of the continuum electron phase in molecular strong-field photoelectron holography," Nat. Phys. 10(8), 594-600 (2014).

16. M. Li et al., "Photoelectron holographic interferometry to probe the longitudinal momentum offset at the tunnel exit," Phys. Rev. Lett. 122(18), 183202 (2019).

17. P. Salières et al., "Feynman's path-integral approach for intenselaser-atom interactions," Science 292(5518), 902-905 (2001).

18. Y. Huismans et al., "Time-resolved holography with photoelectrons," Science 331(6013), 61-64 (2011).

19. X. Xie et al., "Attosecond probe of valence-electron wave packets by subcycle sculpted laser fields," Phys. Rev. Lett. 108(19), 193004 (2012).

20. M. Haertelt et al., "Probing molecular dynamics by laser-induced backscattering holography," Phys. Rev. Lett. 116(13), 133001 (2016).

21. S. Walt et al., "Dynamics of valence-shell electrons and nuclei probed by strong-field holography and rescattering," Nat. Commun. 8(1), 15651 (2017).

22. M. He et al., "Direct visualization of valence electron motion using strong-field photoelectron holography," Phys. Rev. Lett. 120(13), 133204 (2018).

23. X. Lai et al., "Elliptical polarization favors long quantum orbits in high-order above-threshold ionization of noble gases," Phys. Rev. Lett. 110(4), 043002 (2013).

24. M. Möller et al., "Off-axis low-energy structures in above-threshold ionization," Phys. Rev. A 90(2), 023412 (2014).

25. F. Lindner et al., "Attosecond double-slit experiment," Phys. Rev. Lett. 95(4), 040401 (2005).

26. D. Arbó et al., "Interference of electron wave packets in atomic ionization by subcycle sculpted laser pulses," Phys. Rev. A 89(4), 043414 (2014).

27. G. Porat et al., "Attosecond time-resolved photoelectron holography," Nat. Commun. 9(1), 2805 (2018).

28. J. Henkel and M. Lein, "Analysis of electron trajectories with twocolor strong-field ionization," Phys. Rev. A 92(1), 013422 (2015).

29. N. Eicke and M. Lein, "Extracting trajectory information from two-color strong-field ionization," J. Mod. Opt. 64(10-11), 981-986 (2017).

30. N. Eicke et al., "Attosecond-scale streaking methods for strongfield ionization by tailored fields," Phys. Rev. Lett. 124(4), 043202 (2020).

31. M. Richter et al., "Streaking temporal double-slit interference by an orthogonal two-color laser field," Phys. Rev. Lett. 114(14), 143001 (2015)

32. M. Chini et al., "Delay control in attosecond pump-probe experiments," Opt. Express 17(24), 21459-21464 (2009).

33. K. Lin et al., "Directional bond breaking by polarization-gated twocolor ultrashort laser pulses," J. Phys. B 49(2), 025603 (2016).

34. A. Eppink and D. H. Parker, "Velocity map imaging of ions and electrons using electrostatic lenses: application in photoelectron and photofragment ion imaging of molecular oxygen," Rev. Sci. Instrum. 68(9), 3477-3484 (1997). 
35. P. Agostini et al., "Free-free transitions following six-photon ionization of xenon atoms," Phys. Rev. Lett. 42(17), 1127-1130 (1979).

36. Y. Zhou et al., "Near-forward rescattering photoelectron holography in strong-field ionization: extraction of the phase of the scattering amplitude," Phys. Rev. Lett. 116(17), 173001 (2016).

37. S. Zherebtsov et al., "Carrier-envelope phase-tagged imaging of the controlled electron acceleration from $\mathrm{SiO}_{2}$ nanospheres in intense few-cycle laser fields," New J. Phys. 14(7), 075010 (2012).

38. S. Skruszewicz et al., "Two-color strong-field photoelectron spectroscopy and the phase of the phase," Phys. Rev. Lett. 115(4), $043001(2015)$

39. J. Braß et al., "Two-color phase-of-the-phase spectroscopy applied to nonperturbative electron-positron pair production in strong oscillating electric fields," Phys. Rev. A 101(4), 043401 (2020).

40. V. A. Tulsky et al., "Revealing laser-coherent electron features using phase-of-the-phase spectroscopy," J. Phys. B At. Mol. Opt. Phys. 53(7), 074001 (2020).

41. D. Würzler et al., "Accurate retrieval of ionization times by means of the phase-of-the-phase spectroscopy and its limits," Phys. Rev. A 101(3), 033416 (2020).

42. S. Popruzhenko and D. Bauer, "Strong field approximation for systems with Coulomb interaction," J. Mod. Opt. 55(16), 2573-2589 (2008).

43. T. Yan et al., "Low-energy structures in strong field ionization revealed by quantum orbits," Phys. Rev. Lett. 105(25), 253002 (2010).

44. T. Yan and D. Bauer, "Sub-barrier Coulomb effects on the interference pattern in tunneling-ionization photoelectron spectra," Phys. Rev. A 86(5), 053403 (2012).

45. L. Torlina and O. Smirnova, "Coulomb time delays in high harmonic generation," New J. Phys. 19(2), 023012 (2017).

Jia Tan is currently a PhD graduate student with Professor Yueming Zhou at Huazhong University of Science and Technology (HUST), China. Her main research interest is ultrafast dynamics of atoms and molecules in strong laser fields.

Shengliang $\mathbf{X u}$ is a PhD student with Professor Qingbin Zhang at HUST. Before that he received his bachelor's degree in physics from Nanchang
University. His main research interest is ultrafast dynamics in strong laser fields.

Xu Han is a PhD student with Professor Qingbin Zhang at HUST, where he received his bachelor's degree in physics. His main research interest is related to molecules and nanostructures exposed to strong laser fields.

Yueming Zhou is a professor at the School of Physics, HUST, Wuhan, China. He received his $\mathrm{PhD}$ from HUST and did his postdoc as a JSPS research fellow at the University of Electro-Communications. He was selected as an outstanding young scholar of NSFC. His research focuses on the ultrafast electron dynamics of atoms and molecules in strong laser fields.

Min Li is a professor at the School of Physics, HUST, Wuhan, China. $\mathrm{He}$ received his PhD from Peking University in 2015. He was selected as an outstanding young scholar of NSFC. His research focuses on the tunneling ionization of atoms and molecules induced by strong laser fields, quantum control, and molecular dissociation.

Wei Cao is a professor at the School of Physics, HUST, Wuhan, China. He received his $\mathrm{PhD}$ in physics from Kansas State University in 2014. From 2014 to 2017, he worked as a postdoctoral fellow at Lawrence Berkeley National Lab in the United States. His research focuses on the generation of the attosecond pulse and its application for ultrafast process diagnosis.

Qingbin Zhang is a professor at the School of Physics, HUST, Wuhan, China. He received his PhD from HUST in 2011. From 2016 to 2017, he was a visiting scholar at Wayne State University in the United States. His current research interest is related to high-resolution intense field photoelectron full-dimensional detection technology as well as ultrafast optical field manipulation based on the above-mentioned innovative technology.

Peixiang $\mathbf{L u}$ is a professor and the vice director at Wuhan National Laboratory for Optoelectronics, HUST, Wuhan, China. He received his $\mathrm{PhD}$ from Shanghai Institute of Optics and Fine Mechanics. He was selected as a Cheung Kong Scholar Chair Professor, the distinguished young scholar of NSFC, and he was selected as OSA fellow in 2016. His research interest is strong ultrafast optics. 